\title{
A Combined Ab Initio and Bond-Order Potentials Study of Cohesion in Iridium
}

\author{
Marc J. Cawkwell ${ }^{1}$, Duc Nguyen-Manh ${ }^{2}$, Vaclav Vitek ${ }^{1}$ and David G. Pettifor ${ }^{2}$. \\ ${ }^{1}$ Department of Materials Science and Engineering, University of Pennsylvania, \\ 3231 Walnut Street, Philadelphia, PA 19104-6272, U.S.A. \\ ${ }^{2}$ Department of Materials, University of Oxford, Parks Road, \\ Oxford, OX1 3PH, UNITED KINGDOM.
}

\begin{abstract}
The extremely high melting point and excellent resistance to oxidation and corrosion offered by iridium suggest numerous applications of this transition metal in static components at high temperatures and in aggressive environments. However, the mechanical and physical properties of f.c.c. Ir exhibit numerous anomalies when compared to other metals that crystallize in the f.c.c. structure. Notable examples include a negative Cauchy pressure, $1 / 2\left(\mathrm{C}_{12}-\mathrm{C}_{44}\right)$, brittle transgranular cleavage after a period of plastic flow even in pure single crystals and anomalous

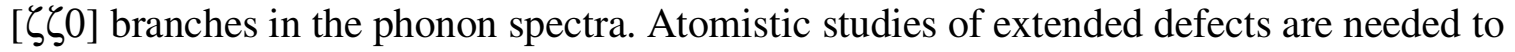
elucidate the origin of anomalous mechanical properties, such as brittleness. For this purpose we developed a Bond-Order Potential (BOP), an $O(N)$ tight-binding formalism, employing physically transparent parameterizations that use experimental and ab initio data, generated in this study using the Full Potential Augmented Plane Wave plus Local Orbitals (APW+lo) method. The constructed BOP reproduces then both equilibrium as well as a variety of nonequilibrium properties of Ir and represents an excellent description of cohesion in f.c.c. Ir. This description of interatomic interactions is imminently suitable for studies of defects, such as dislocations and grain boundaries, that control plastic deformation and fracture.
\end{abstract}

\section{INTRODUCTION}

An unusual combination of excellent physical properties, such as extremely high melting temperature $\left(2442{ }^{\circ} \mathrm{C}\right)$ and corrosion resistance, and properties that are exceptional for metals with the f.c.c. structure, have long since highlighted iridium as worthy of significant industrial interest and academic study. Owing to its excellent high temperature properties, a class of iridium based superalloys have been advanced [1] and a number conventional alloys with iridium as basis have been developed [2]. Despite these advances in the synthesis of industrially important refractory alloys, the basic understanding of mechanical properties of pure Ir is still only rudimental.

The most significant anomalous property, detrimental to more widespread applications of this transition metal, is its tendency to undergo brittle cleavage under tensile loading [3,4]. This behavior persists in high purity single crystals, suggesting that it is an intrinsic property of f.c.c. $\operatorname{Ir}[4,5]$. Iridium can undergo brittle transgranular cleavage at temperatures up to $950{ }^{\circ} \mathrm{C}$, and after significant plastic deformation [6].

Iridium is also unique amongst the cubic transition metals in that it has a negative Cauchy pressure, $1 / 2\left(\mathrm{C}_{12}-\mathrm{C}_{44}\right)$. This is a strong indication that the interatomic bonding in Ir has a significant non-central character and is a characteristic of materials that exhibit mixed metallic and covalent bonding, such as transition metal aluminides [7]. Another indication of a strong 
non-central character to interatomic bonding in iridium is the pronounced anomalies in its

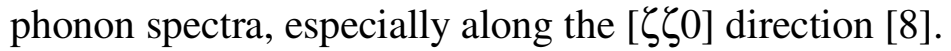

In order to generate a reliable and transferable model of interatomic interactions in iridium that would be suitable for the atomistic simulation of extended defects, it is essential to capture the non-central bonding character arising from the unsaturated $5 d$ band. Therefore, many-body central force schemes, such as the Embedded Atom Method [9] or Finnis-Sinclair potentials [10], are not suited to our purpose. Instead, we have adopted the Bond-Order Potential formalism advanced by Pettifor and coworkers, see for example [11, 12]. BOPs are a real-space, $O(N)$ scaling tight-binding based framework that naturally describes systems with mixed metallic and covalent bonding $[7,13]$. Recent advances in the implementation of BOPs to the study of materials that exhibit negative Cauchy pressures [7, 14, 15] allow us to fit the Cauchy pressure through a physically transparent, environmentally dependent many-body repulsive term in the total energy.

In this paper, we briefly outline the BOP formalism and present results of the testing of the recently constructed BOP for Ir. Extensive testing has been performed in order to evaluate its efficacy as a description of cohesion in f.c.c. Ir, along with its transferability to structures that deviate significantly from the ideal f.c.c. environment. In particular, we have calculated the energies of the intrinsic and extrinsic stacking faults and the twin boundary as well as the vacancy formation energy. Results of these calculations are compared with experimental and $a b$ initio results. We have also studied the dependence of structural energy differences (SEDs) on atomic volume for a number of alternate structures using both BOP and $a b$ initio methods. In addition, the trigonal and tetragonal deformation paths have been investigated and compared with $a b$ initio results. Finally, we have calculated the phonon spectra of f.c.c. Ir for three high symmetry directions using frozen phonons and compared our results with experimental observations.

\section{THEORY}

The BOP formalism has been extensively documented, see for example $[7,12,13]$, and so we present only a brief recap here. Starting from the Tight Binding Bond model of Sutton et al. [16] we write $E_{\text {tot }}=E_{\text {bond }}+E_{\text {pair. }}$. Within BOP, $E_{\text {bond }}$ takes the form,

$$
E_{b o n d}=\sum_{\substack{i \neq j \\ \alpha, \beta}} \Theta_{j \beta, i \alpha} H_{i \alpha, j \beta}
$$

where $\Theta_{j \beta, i \alpha}$ is the bond order and $H_{i \alpha, j \beta}$ is the Hamiltonian matrix; summation extends over all atoms $i$ and $j$ and orbitals $\alpha$ and $\beta$. The angular dependence of the hopping integrals is given by the usual Slater and Koster form, whilst their radial dependence is represented by the Goodwin, Skinner, Pettifor (GSP) function [17]. We fit the GSP function to hopping integrals evaluated using the orbital downfolding technique to TB-LMTO data. The radial part of the bond term is augmented with a polynomial of fifth order beyond a certain radius to allow for a smooth decrease to zero at a defined distance, in our case, between second and third nearest neighbors in the f.c.c. lattice. The onsite elements of the Hamiltonian matrix are adjusted self-consistently during a simulation to ensure that the condition of local charge neutrality is enforced. We achieve a real-space, $O(N)$ scheme through dispensing with the direct diagonalization of the Hamiltonian matrix, utilizing instead the concept of reconstructing the local densities of states 
through the moments theorem of Ducastelle and Cyrot-Lackmann [18], by employing the Lanczos recursion method of continuous fractions [19]

The Cauchy pressures given by the bond part are generally in poor agreement with experimental and/or $a b$ initio results and are always positive. Nguyen-Manh [7, 14, 15] found that the negative Cauchy pressures in certain materials originates in a many-body repulsive term, which represents the overlap repulsion experienced by the $s p$-electrons when compressed by the bonding contribution from the $d$-electrons. Hence, we extended our description of the total energy with BOP to include this environmentally dependent many-body repulsive term, i.e., $E_{\text {tot }}=E_{\text {bond }}+E_{\text {pair }}+E_{\text {env }}$. This term assumes the form of a screened Yukawa potential [7],

$$
E_{e n v}=1 / 2 \sum_{i \neq j} B \frac{\exp \left[-\lambda\left(R_{i j}-2 R_{c}\right)\right]}{R_{i j}} \quad \text { where } \lambda=\lambda_{0}+\left[\sum_{k \neq i} C \exp \left(-v R_{i k}\right)\right]^{1 / \gamma}
$$

and $B, C, v, \lambda_{0}$ and $\gamma$ are adjustable parameters. $R_{c}$ is related to the radius of the valence $s$ electron states.

The environmentally dependent many-body repulsive term allows us to fit iridium's negative Cauchy pressure exactly. We then use the pairwise term, which represents the contribution to cohesion from the free electron-like $s p$-band in transition metals, to fit the remaining elastic constants, along with the lattice parameter of f.c.c. Ir and its cohesive energy. The pairwise interaction in our BOP scheme takes the same form as in [7]:

$$
E_{\text {pair }}=1 / 2 \sum_{i \neq j} \sum_{k=1}^{4} A_{k}\left(R_{k}-R_{i j}\right)^{3} H\left(R_{k}-R_{i j}\right)
$$

The node points, $R_{k}$ and $A_{k}$ are used as fitting parameters and $H(x)$ is the Heaviside step function. There is substantial freedom in the choice of the node points, allowing us to tune the pairwise term so that the BOP can also reproduce energy differences calculated ab initio. Specifically, for Ir, the pairwise term was used to reproduce the ideal h.c.p. - f.c.c. SED, along with the position of the minimum in the dependence of the ideal h.c.p. Ir total energy on volume.

The ability of any semi-empirical model to reliably describe cohesion in a given material is dependent on the parameterization employed. It is therefore necessary to have a large database of experimental and $a b$ initio data, both for determining a parameterization and its subsequent testing. For this purpose, we performed a series of $a b$ initio electronic structure calculations within the Density Functional Theory. We carried out calculations using the FLAPW and APW+lo methods employing the WIEN2k [20] package of programs. In both cases, calculations were made within the generalized gradient approximation, using the exchange-correlation functional of Perdew, Burke and Ernzerhof [21]. Our FLAPW and APW+lo calculations gave identical total energies to within $1.0 \mathrm{mRy} /$ atom for all structures studied, but with the APW+lo calculations being considerably less computationally demanding.

\section{RESULTS}

When constructing our BOP for Ir only the contribution of $d$-electrons was included into $E_{\text {bond }}$. The recursion algorithm was applied to 4 levels, including thus the first 7 moments of the densities of states. TB-LMTO results indicated that f.c.c. Ir possesses 7.1 electrons of $d$-character per atom, and this value has been used in the BOP. It is important to note that with this filling of the $d$-band, the bond part predicts that h.c.p. Ir has lower energy than the f.c.c. structure by $27.5 \mathrm{meV} /$ atom. This picture is consistent with previous studies of the structural stability of the 
transition metals using $d$-interactions only [22]. Increasing the number of recursion levels does not change the sign of this SED. The other two terms in the total energy favor f.c.c. over h.c.p. strongly, therefore producing a BOP that predicts the correct order of structural stability.

In Figure 1, SEDs vs. atomic volume are plotted, calculated using both BOP and APW+lo. We see that our BOP predicts the correct order of structural stability, i.e., f.c.c. - h.c.p. - A15 b.c.c. - s.c. It should be noted that both APW+lo and FLAPW calculations indicate that b.c.c. Ir is ferromagnetic, with a magnetic moment of $0.481 \mu_{\mathrm{B}}$ per unit cell. The results of spin (sp_APW+lo) and non-spin polarized calculations for b.c.c. Ir are shown in Figure 1.

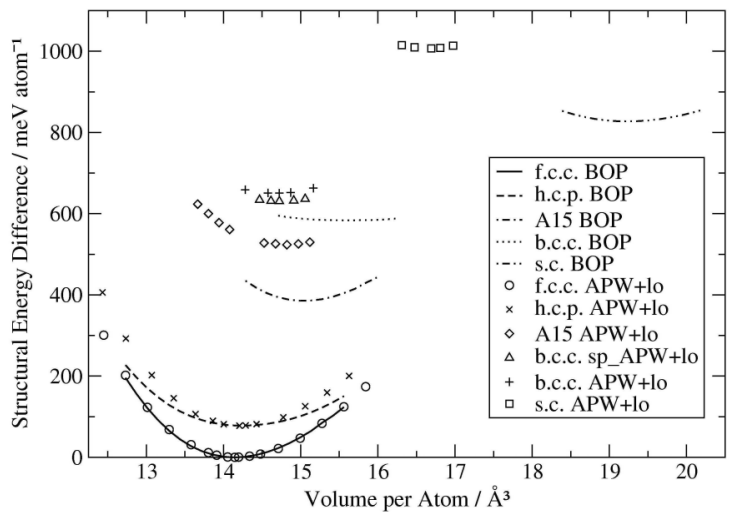

Figure 1. Structural energy differences vs. atomic volume for f.c.c., h.c.p., A15, b.c.c. and s.c. Ir calculated from BOP and APW+lo. The APW+lo values have been normalized to the experimental volume per atom. sp_APW+lo denotes a spin polarized calculation

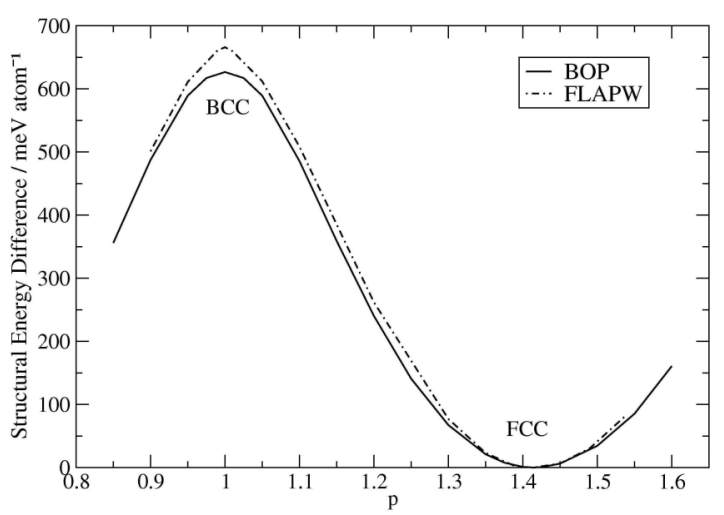

Figure 2. Tetragonal deformation path calculated using BOP and FLAPW

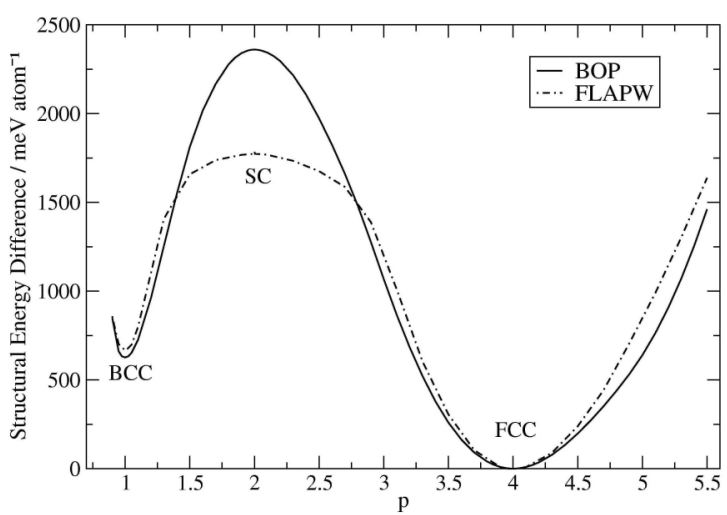

Figure 3. Trigonal defomation path calculated using BOP and FLAPW

In Figures 2 and 3 we compare for the tetragonal and trigonal deformation paths the dependencies of the energy on a parameter $\mathrm{p}$ (which defines these paths) evaluated using our BOP to those calculated using FLAPW. Readers not familiar with such deformation paths are directed to [23]. It is evident from Figures 1, 2 and 3 that BOP, which is fitted to the properties of close-packed structures only, is transferable to the more open b.c.c. and A15 structures, as well as to less symmetric structures encountered along the deformation paths. However, we find that the equilibrium volume of s.c. Ir is overestimated by $12 \%$ in comparison with ab initio prediction. The description of more open structures could be improved through the application of a screening function to the hopping integrals $[12,24]$ to better describe the second nearest neighbor interactions in these structures, but we feel that the extra computational effort required does not warrant this relatively small enhancement.

The phonon spectra of f.c.c. Ir were calculated using the method of frozen phonons along

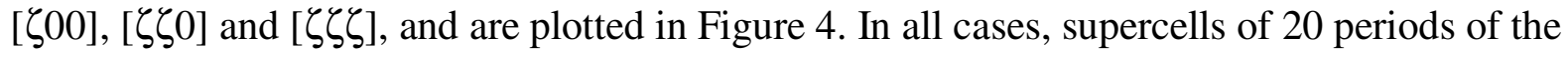


underlying lattice were constructed and the amplitude of the applied waves were $0.02 \AA$. The experimental phonon spectra are reported in [8].

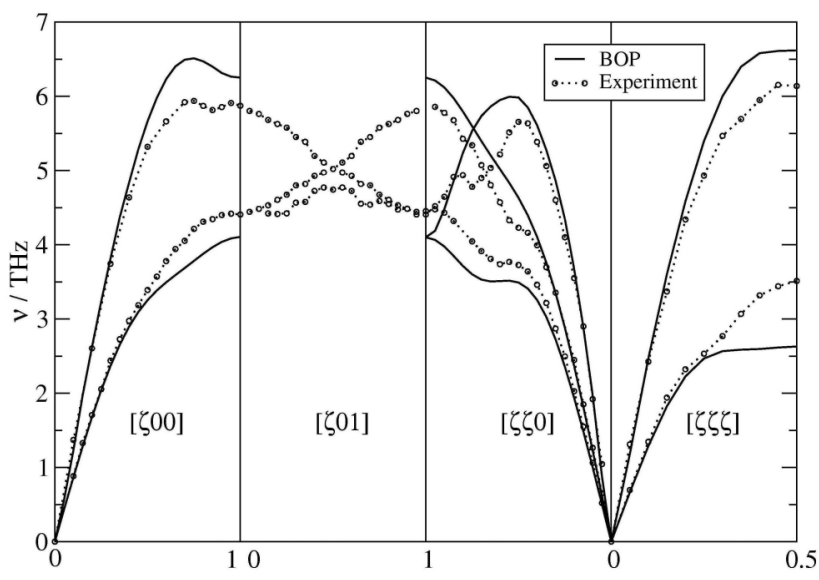

Figure 4. Phonon spectra of f.c.c. iridium calculated using BOP and compared with experimental results.

Figure 4 clearly illustrates that our BOP for Ir reproduces accurately the phonon spectra, and

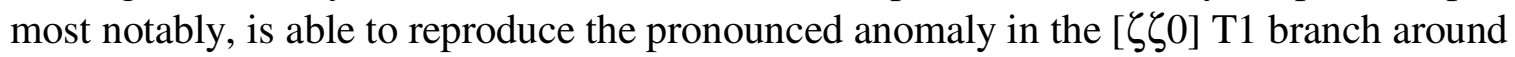
$\zeta=0.5$. We believe the soft transverse $[\zeta \zeta \zeta]$ branches are a consequence of the bond part of the BOP favoring h.c.p. over f.c.c. as these phonon modes correspond to a shearing of $\{111\}$ planes parallel to each other.

The energies of several lattice defects in f.c.c. iridium have been calculated using a supercell approach. In these simulations, the cells were relaxed until the force acting on any atom was less than $0.01 \mathrm{eV}^{-1}$. The energy of the intrinsic stacking fault was found to be $407.8 \mathrm{~mJ} \mathrm{~m}^{-2}$, in excellent agreement with experiment, $420 \mathrm{~mJ} \mathrm{~m}^{-2}$ [25] and ab initio calculation, $365 \mathrm{~mJ} \mathrm{~m}^{-2}$ [26]. We also calculated the energies of the extrinsic stacking fault and the twin boundary. They are $461.9 \mathrm{~mJ} \mathrm{~m}^{-2}$ and $221.8 \mathrm{~mJ} \mathrm{~m}^{-2}$ respectively; as expected the energies of the intrinsic and extrinsic stacking faults are similar and the energy of the twin boundary is about half of the energy of the stacking faults. The vacancy formation energy predicted by our model is $2.97 \mathrm{eV}$, which overestimates the results of ab initio calculations [26, 27] by about $0.8 \mathrm{eV}$.

\section{CONCLUSIONS}

We have presented a BOP for iridium that reproduces accurately its elastic constants, and negative Cauchy pressure, through the introduction of a many-body repulsive term in the total energy. This BOP displays very good transferability to structures substantially different to the f.c.c. environment and can accurately predict the energies of a number of crystal defects. It is also able to reproduce phonon spectra of f.c.c. Ir, including certain anomalous features. With such an accurate description of cohesion, we believe we will be able to employ this BOP in studies of the atomic structure and properties of extended defects in f.c.c. Ir with confidence.

\section{ACKNOWLEDGEMENTS}

The authors are grateful to Dr. R. Heid and Professor M. Sob for supplying tabulated data for the phonon spectra and deformation paths, respectively. M.J.C. thanks Dr. M. Mrovec for 
many useful discussions on BOPs. This work was funded in part (M.J.C. and V.V.) by the U.S. Department of Energy, BES Grant no. DE-FG02-98ER45702.

\section{REFERENCES}

1. Y. Yamabe-Mitari, Y. Ro, T. Makuro and H. Harada. Metall. Mater. Trans. 29A, 537 (1998)

2. C.G. McKamey, E.P. George, E.H. Lee, E.K. Ohriner, J. Heatherly and J.W. Cohron. Scripta Mater. 42, 9 (2000)

3. P. Panfilov, A. Yermakov, V. Dmitriev and N. Timofeev. Platinum Metals Rev. 35, 196 (1991)

4. A. Yermakov, P. Panfilov and R. Adamesku. J. Mat. Sci. Letts. 9, 696 (1990)

5. S.S. Hecker, D.L. Rohr and D.F. Stein. Metall. Trans. A. 9, 481 (1978)

6. P. Panfilov, V. Novgorodov and A. Yermakov. J. Mat. Sci. Letts. 14, 137 (1994)

7. S. Znam, D. Nguyen-Manh, D.G. Pettifor and V. Vitek. Phil. Mag. A. 83, 415 (2003)

8. R. Heid, K-P Bohnen, K. Felix, K.M. Ho and W. Reichardt. J. Phys.: Condens. Matter. 10, 7967 (1998)

9. M.S. Daw and M.I. Baskes. Phys. Rev. B. 29, 6443 (1984)

10. M.W. Finnis and J.E. Sinclair. Phil. Mag. A. 50, 45 (1984)

11. A.P. Horsfield, A.M. Bratkovsky, M. Fearn, D.G. Pettifor and M. Aoki. Phys. Rev. B. 53, 12694 (1996)

12. M. Mrovec. PhD. Thesis. University of Pennsylvania (2002)

13. A. Girshick, A.M. Bratkovsky, D.G. Pettifor and V. Vitek. Phil. Mag. A. 77, 981 (1998)

14. D. Nguyen-Manh, D.G. Pettifor, S. Znam and V. Vitek. Mat. Res. Soc. Symp. Proc. Vol. 491, 353 (1998)

15. D. Nguyen-Manh, D.G. Pettifor, D.J.H. Cockayne, M. Mrovec, S. Znam and V. Vitek. Bull. Mater. Sci. 26, 43 (2003)

16. A.P. Sutton, M.W. Finnis, D.G. Pettifor and Y. Ohta. J. Phys. C.: Solid State Phys. 21, 35 (1988)

17. L. Goodwin, A.J. Skinner and D.G. Pettifor. Europhys. Letts. 9, 701 (1989)

18. F. Ducastelle and F. Cyrot-Lackmann. J. Phys. Chem. Sol. 31, 1295 (1970)

19. C. Lanczos. J. Res. Natl. Bur. Stand. 45, 225 (1950)

20. P. Blaha, K. Schwarz, G.K.H. Madsen, D. Kvasnicka and J. Luitz, WIEN2k, An Augmented Plane Wave + Local Orbitals Program for Calculating Crystal Properties (Karlheinz Schwarz, Techn. Universität Wien, Austria), 2001. ISBN 3-9501031-1-2.

21. J.P. Perdew, K. Burke and M. Ernzerhof. Phys. Rev. Letts. 77, 3865 (1996)

22. H.L. Skriver. Phys. Rev. B. 31, 1909 (1985)

23. V. Paidar, L.G. Wang, M. Sob and V. Vitek. Modelling Simul. Mater. Sci. Eng. 7, 369 (1999)

24. D. Nguyen-Manh, D.G. Pettifor and V. Vitek. Phys. Rev. Letts. 85, 4136 (2000)

25. T.J. Balk and K.J. Hemker. Phil. Mag. A. 81, 1507 (2001)

26. Yu.N. Gornostyrev, M.I. Katsnelson, N.I. Medvedeva, O.N. Mryasov, A.J. Freeman and A.V. Trefilov. Phys. Rev. B. 62, 7802 (2000)

27. P.A. Korzhavyi, I.A. Abrikosov, B. Johansson, A.V. Ruban and H.L. Skriver. Phys. Rev. B. 59, 11693 (1999) 